\title{
Modelling, design and analysis of three controllers based on LQR formulation for a non-linear hydraulic uniaxial seismic shake table
}

\author{
José Luis Sarmiento and Carlos Borrás Pinilla \\ Mechanical Engineering Faculty, Universidad Industrial de Santander, 680002 Bucaramanga, Colombia
}

\begin{abstract}
This study presents the modelling, design and analysis of three controllers applied to the non-linear model of a hydraulic uniaxial seismic shake table. Firstly, the system's non-linear model is constructed based on the dynamic and mathematical analysis of the hydraulic actuator and the servo valve. Then, three control systems based on the LQR formulation are designed for the acceleration tracking: Linear quadratic integral (LQI), linear quadratic tracking (LQT) and a variation of LQT. Lastly, simulations are carried out using the non-linear model as the plant and the results showed that the variation of the LQT control exhibited the best acceleration tracking performance.
\end{abstract}

\section{Introduction}

The worldwide seismic activity has given engineers the responsibility to study, analyse and understand seismological phenomena to develop structures more resistant to earthquakes in order to saves as many lives as possible during a catastrophe of this nature [1]. Seismic shake tables are one of the tools developed jointly by mechanic, electronic and civil engineers to study the dynamic behaviour of structures subject to earthquakes [2]. The main objective of a seismic table is to reproduce reference earthquake accelerations on test structures [3].

Hydraulic seismic shake tables are the most popular since they allow greater load capacity, frequency and motion amplitude. However, acceleration tracking control is extremely complicated due to the non-linearities inherent to hydraulic systems and the existence of an unstable and unobservable mode in its measurement [3].

In this work the non-linear dynamic model was first obtained for the hydraulic uniaxial seismic shake table. Then, three controllers based on the LQR formulation were designed: Linear quadratic integral (LQI), linear quadratic tracking (LQT) and a variation of LQT. Finally, simulations were carried out to compare the tracking performance of the proposed controllers and conclusions were established for a later experimental implementation in the seismic table of the Dynamics and Structural Control Lab at Universidad Industrial de Santander.

\section{System dynamics}

The schematic representation of the uniaxial seismic table hydraulic system under consideration in shown in Fig 1.
The hydraulic actuator is double rod and is commanded by a servo valve. The system dynamics is established based on the analysis of this two elements [4].

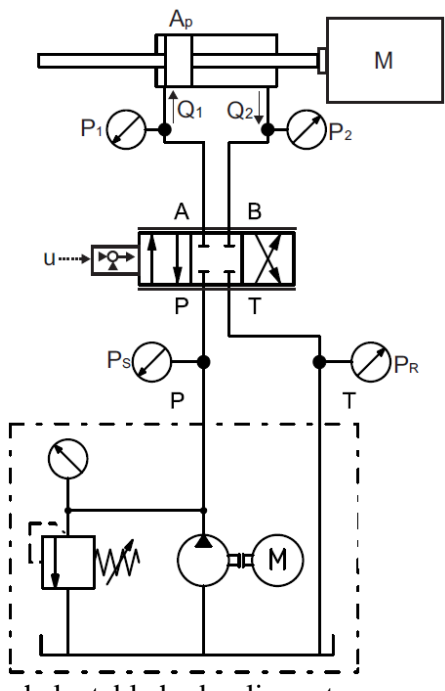

Fig 1. Seismic shake table hydraulic system.

\subsection{Actuator analysis}

The motion dynamics of the seismic shake table platform is obtained applying Newton's second law of motion

$$
M \ddot{x}_{p}=A_{p} P_{L}-C \dot{x}_{p}-K x_{p}
$$

where $M$ is the platform mass, $x_{p}$ the horizontal platform displacement, $C$ the viscous damping coefficient, $K$ the mass stiffness, $A_{p}$ the effective piston area and $P_{L}$ the load pressure, defined as $P_{L}=P_{1}-P_{2}$. 
The actuator's dynamics is obtained applying the continuity principle in each chamber and neglecting leakage

$$
Q_{1}=\frac{d V_{1}}{d t}+\frac{V_{1}}{\beta_{1 e}} \frac{d P_{1}}{d t}, \quad-Q_{2}=\frac{d V_{2}}{d t}+\frac{V_{2}}{\beta_{2 e}} \frac{d P_{2}}{d t}
$$

where $Q_{1}$ is the flow into chamber $1, Q_{2}$ the flow into chamber $2, V_{1}$ and $V_{2}$ the volume of chambers 1 and 2 respectively, $\beta_{1 e}$ and $\beta_{2 e}$ the effective bulk modulus in chambers 1 and 2 respectively, and $P_{1}$ and $P_{2}$ the pressures in each chamber [5]. Defining the relationship between the chamber volumes and the actuator position gives

$$
V_{1}=V_{o 1}+A_{p} x_{p}, \quad V_{2}=V_{o 2}-A_{p} x_{p}
$$

where $V_{o 1}$ and $V_{o 2}$ are the constant equilibrium values for the volume in each chamber. Differentiating equations 3 yields the relations between velocity and volume change

$$
\frac{d V_{1}}{d t}=A_{p} \dot{x}_{p}, \quad \frac{d V_{2}}{d t}=-A_{p} \dot{x}_{p}
$$

Defining the load flow as $Q_{L}=\left(Q_{1}+Q_{2}\right) / 2$, and combining equations 2 and 4 gives [5].

$$
Q_{L}=A_{p} \dot{x}_{p}+\frac{V_{1}}{2 \beta_{1 e}} \frac{d P_{1}}{d t}-\frac{V_{2}}{2 \beta_{2 e}} \frac{d P_{2}}{d t}
$$

Assuming that the bulk modulus is the same for both chambers, equation 5 is approximated to equation 6 where $\beta$ is the effective Bulk Modulus and $V_{T}$ the total fluid volume trapped in actuator's chambers and pipes.

$$
Q_{L}=A_{p} \dot{x}_{p}+\frac{V_{T}}{4 \beta} \dot{P}_{L}
$$

\subsection{Servo valve analysis}

The flow supplied by the servo valve is related to the spool displacement by equation 7 (assuming symmetric orifices), where $C_{d}$ is the discharge coefficient through the orifice, $w$ the area gradient of the orifice, $x_{v}$ the spool displacement, $P_{S}$ the supply pressure and $\rho$ the hydraulic fluid density. This equation is linearized using a firstorder Taylor expansion to obtain equation 8, where $K_{f}$ and $K_{P}$ are known as flow gain and pressure-flow coefficient respectively [4].

$$
\begin{gathered}
Q_{L}=C_{d} w x_{v} \sqrt{\frac{P_{S}-\operatorname{sign}\left(x_{v}\right) P_{L}}{\rho}} \\
Q_{L}=K_{f} x_{v}-K_{P} P_{L} \\
K_{f}=C_{d} w \sqrt{\frac{P_{S}-P_{L o}}{\rho}}, \quad K_{P}=\frac{C_{d} w x_{v o}}{2 \sqrt{\rho\left(P_{S}-P_{L o}\right)}}
\end{gathered}
$$

The control voltage and spool displacement relationship is defined by the second-order differential equation 10 where $k_{v}, \zeta_{v}$ and $\omega_{v}$ are the gain, damping coefficient and natural frequency of the servo valve, and $u$ is the control voltage [6].

$$
u=\frac{1}{k_{v}}\left(\frac{1}{\omega_{v}^{2}} \ddot{x}_{v}+\frac{2 \zeta_{v}}{\omega_{v}} \dot{x}_{v}+x_{v}\right)
$$

\subsection{Non-linear state equations}

Using equations $1,6,7$ and 10 , and defining the state variables as $x_{1}=x_{p}, x_{2}=\dot{x}_{p}, x_{3}=P_{L}, x_{4}=x_{v}, x_{5}=\dot{x}_{v}$, the non-linear state equations are built as follows

$$
\begin{gathered}
\dot{x}_{1}=x_{2} \\
\dot{x}_{2}=\frac{1}{M}\left(A_{p} x_{3}-C x_{2}-K x_{1}\right) \\
\dot{x}_{3}=\frac{4 \beta}{V_{T}}\left(C_{d} w x_{4} \sqrt{\frac{P_{S}-x_{3}}{\rho}}-A_{p} x_{2}\right) \\
\dot{x}_{4}=x_{5} \\
\dot{x}_{5}=\omega_{v}^{2}\left(k_{v} u-x_{4}-\frac{2 \zeta_{v}}{\omega_{v}} x_{5}\right)
\end{gathered}
$$

This equations define the non-linear model of the hydraulic uniaxial seismic shake table. However, in order to design the proposed controllers it is necessary to obtain the linearized version of this equations and construct the state-space representation.

\subsection{Linear state equations and state-space representation}

Using equations 11 to 15 and the linearized equation of the flow supplied by the servo valve (equation 8 ), the linear state equations are built as follows

$$
\begin{gathered}
\dot{x}_{1}=x_{2} \\
\dot{x}_{2}=\frac{1}{M}\left(A_{p} x_{3}-C x_{2}\right) \\
\dot{x}_{3}=\frac{4 \beta}{V_{T}}\left(K_{f} x_{4}-K_{P} x_{3}-A_{p} x_{2}\right) \\
\dot{x}_{4}=x_{5} \\
\dot{x}_{5}=\omega_{v}^{2}\left(k_{v} u-x_{4}-\frac{2 \zeta_{v}}{\omega_{v}} x_{5}\right)
\end{gathered}
$$

Based on the state equations the state-space representation of the linearized single-input single-output (SISO) system of the form $\dot{\boldsymbol{x}}=\boldsymbol{A} \boldsymbol{x}+\boldsymbol{B} \boldsymbol{u}$ with output equation $\boldsymbol{y}=\boldsymbol{C} \boldsymbol{x}$ is constructed

$$
\begin{gathered}
{\left[\begin{array}{c}
\dot{x}_{1} \\
\dot{x}_{2} \\
\dot{x}_{3} \\
\dot{x}_{4} \\
\dot{x}_{5}
\end{array}\right]=\left[\begin{array}{ccccc}
0 & 1 & 0 & 0 & 0 \\
\frac{-K}{M} & \frac{-C}{M} & \frac{A_{p}}{M} & 0 & 0 \\
0 & \frac{-4 \beta A_{p}}{V_{T}} & \frac{-4 \beta K_{P}}{V_{T}} & \frac{4 \beta K_{f}}{V_{T}} & 0 \\
0 & 0 & 0 & 0 & 1 \\
0 & 0 & 0 & -\omega_{v}^{2} & -2 \zeta_{v} \omega_{v}
\end{array}\right]\left[\begin{array}{l}
x_{1} \\
x_{2} \\
x_{3} \\
x_{4} \\
x_{5}
\end{array}\right]+\left[\begin{array}{c}
0 \\
0 \\
0 \\
0 \\
k_{v} \omega_{v}^{2}
\end{array}\right] u} \\
y=\left[\begin{array}{lllll}
1 & 0 & 0 & 0 & 0
\end{array}\right]\left[\begin{array}{l}
x_{1} \\
x_{2} \\
x_{3} \\
x_{4} \\
x_{5}
\end{array}\right]
\end{gathered}
$$

\section{Control design}

Three control strategies based on the LQR formulation were proposed and designed for the control of the uniaxial seismic shake table. The first one is the linear 
quadratic integral (LQI) control, which includes all the system's states and the tracking error in the cost function. The second one is the linear quadratic tracking (LQT) control, which only includes the tracking error in the cost function, and the third one is a variation of the LQT that includes the tracking error and its derivative in the cost function.

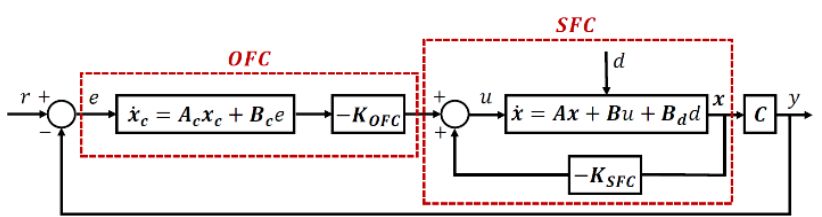

Fig 2. Closed-loop structure of the proposed controller.

Generally the closed-loop structure of the proposed controllers consists of a state-feedback controller (SFC) and an output feedback controller (OFC) as shown in Fig 2 [7] [8]. The linear single-input single-output (SISO) system state-space representation of the plant is defined as

$$
\dot{\boldsymbol{x}}=\boldsymbol{A} \boldsymbol{x}+\boldsymbol{B} u+\boldsymbol{B}_{\boldsymbol{d}} d, \quad y=\boldsymbol{C} \boldsymbol{x}
$$

where $d$ is the disturbance signal and $\boldsymbol{B}_{\boldsymbol{d}}$ the disturbance vector. The structure of the OFC is specified based on the internal model (IM) principle and takes the tracking error $e$ as the input [9]. The OFC state-space representation is

$$
\dot{x}_{c}=A_{c} x_{c}+B_{c} e
$$

Defining the new state vector as $\boldsymbol{x}_{a}=\left[\begin{array}{ll}\boldsymbol{x}^{T} & \boldsymbol{x}_{c}^{T}\end{array}\right]^{T}$ then the augmented system is

$$
\begin{gathered}
\dot{\boldsymbol{x}}_{a}=\boldsymbol{A}_{\boldsymbol{a}} \boldsymbol{x}_{\boldsymbol{a}}+\boldsymbol{B}_{\boldsymbol{a}} u+\boldsymbol{B}_{r} r+\boldsymbol{B}_{\boldsymbol{a d}} d \\
y=\boldsymbol{C}_{\boldsymbol{a}} \boldsymbol{x}_{\boldsymbol{a}}
\end{gathered}
$$

where the augmented matrices are

$$
\begin{gathered}
\boldsymbol{A}_{\boldsymbol{a}}=\left[\begin{array}{cc}
\boldsymbol{A} & \mathbf{0} \\
-\boldsymbol{B}_{c} \boldsymbol{C} & \boldsymbol{A}_{c}
\end{array}\right], \quad \boldsymbol{B}_{a}=\left[\begin{array}{c}
\boldsymbol{B} \\
0
\end{array}\right], \quad \boldsymbol{C}_{a}=\left[\begin{array}{ll}
\boldsymbol{C} & 0
\end{array}\right] \\
\boldsymbol{B}_{r}=\left[\begin{array}{c}
0 \\
\boldsymbol{B}_{c}
\end{array}\right], \quad \boldsymbol{B}_{a d}=\left[\begin{array}{c}
\boldsymbol{B}_{d} \\
0
\end{array}\right]
\end{gathered}
$$

The control input for this system is defined by equation 29 where the gain matrix $\boldsymbol{K}$ consists of the statefeedback gain $\boldsymbol{K}_{\boldsymbol{S}}$ and the output feedback gain $\boldsymbol{K}_{\boldsymbol{O}}$.

$$
u=-K x_{a}=-\left[\begin{array}{l}
K_{S} \\
K_{o}
\end{array}\right]\left[\begin{array}{ll}
x & x_{c}
\end{array}\right]=-K_{S} x-K_{o} x_{c}
$$

\subsection{Linear quadratic integral (LQI) control}

In the LQI control the structure of the OFC is selected as a simple first-order integrator. Thus its state-space representation is defined as $\dot{x}_{c}=e$, where $e$ is the tracking error, defined as the difference between the reference $r$ and the output $y$. The state-space representation of the augmented system is transformed by replacing the OFC representation in equation 25

$$
\left[\begin{array}{c}
\dot{\boldsymbol{x}} \\
\dot{x}_{c}
\end{array}\right]=\left[\begin{array}{cc}
\boldsymbol{A} & \mathbf{0} \\
-\boldsymbol{C} & 0
\end{array}\right]\left[\begin{array}{c}
\boldsymbol{x} \\
x_{c}
\end{array}\right]+\left[\begin{array}{c}
\boldsymbol{B} \\
0
\end{array}\right] u+\left[\begin{array}{l}
0 \\
1
\end{array}\right] r+\left[\begin{array}{c}
\boldsymbol{B}_{\boldsymbol{d}} \\
0
\end{array}\right] d
$$

$$
y=\left[\begin{array}{ll}
\boldsymbol{C} & 0
\end{array}\right]\left[\begin{array}{c}
\boldsymbol{x} \\
x_{c}
\end{array}\right]
$$

The objective of the LQI control is to determine the gain matrix $\boldsymbol{K}$ of the optimal control law defined in equation 30 that minimizes the cost function

$$
J=\int_{0}^{\infty}\left(\boldsymbol{x}_{\boldsymbol{a}}^{T} \boldsymbol{Q} \boldsymbol{x}_{\boldsymbol{a}}+u^{T} R u\right) d t
$$

where $\boldsymbol{Q}$ is the penalty matrix of all the system's states and the tracking error defined as shown in equation 33, where $q_{1}$ is the penalty of state $x_{1}, q_{2}$ the penalty of state $x_{2}$, and so on, until $q_{e}$ which is the penalty of the tracking error. And $R$ is the scalar penalty of the control signal.

$$
\boldsymbol{Q}=\left[\begin{array}{cccccc}
q_{1} & 0 & 0 & 0 & 0 & 0 \\
0 & q_{2} & 0 & 0 & 0 & 0 \\
0 & 0 & q_{3} & 0 & 0 & 0 \\
0 & 0 & 0 & q_{4} & 0 & 0 \\
0 & 0 & 0 & 0 & q_{5} & 0 \\
0 & 0 & 0 & 0 & 0 & q_{e}
\end{array}\right]
$$

By replacing the control law in equation 25 the statespace representation of the augmented system for the LQI control is obtained.

$$
\left[\begin{array}{l}
\dot{\boldsymbol{x}} \\
\dot{x}_{c}
\end{array}\right]=\left[\begin{array}{cc}
\boldsymbol{A}-\boldsymbol{B} \boldsymbol{K}_{\boldsymbol{S}} & -\boldsymbol{B} \boldsymbol{K}_{\boldsymbol{o}} \\
-\boldsymbol{C} & 0
\end{array}\right]\left[\begin{array}{l}
\boldsymbol{x} \\
x_{c}
\end{array}\right]+\left[\begin{array}{l}
0 \\
1
\end{array}\right] r+\left[\begin{array}{c}
\boldsymbol{B}_{\boldsymbol{d}} \\
0
\end{array}\right] d
$$

\subsection{Linear quadratic tracking (LQT) control}

In the LQT control the structure of the OFC is selected as a simple first-order integrator as in the LQI control. However, the objective of this control is to determine the gain matrix $\boldsymbol{K}$ of the control law defined by equation 29 that minimizes the cost function

$$
J=\int_{0}^{\infty}\left(q e^{2}+v^{2}\right) d t
$$

where $q$ is the scalar penalty of the tracking error and $v$ is a transformed input. In this formulation, the cost function is only penalizing the tracking error instead of all the system's states plus the tracking error as in the LQI.

The solution of the LQT problem is done using the LQR formulation if the desired cost is transformed to

$$
J=\int_{0}^{\infty}\left(\boldsymbol{x}_{\boldsymbol{a}}^{\boldsymbol{T}} \boldsymbol{Q} \boldsymbol{x}_{\boldsymbol{a}}+v^{2}\right) d t
$$

and the penalty matrix $\boldsymbol{Q}$ is selected as shown in equation 37 , which is full of zeroes and only includes the penalty of the tracking error $q_{e}$ in the last place.

$$
\boldsymbol{Q}=\left[\begin{array}{llllll}
0 & 0 & 0 & 0 & 0 & 0 \\
0 & 0 & 0 & 0 & 0 & 0 \\
0 & 0 & 0 & 0 & 0 & 0 \\
0 & 0 & 0 & 0 & 0 & 0 \\
0 & 0 & 0 & 0 & 0 & 0 \\
0 & 0 & 0 & 0 & 0 & q_{e}
\end{array}\right]
$$

\subsection{LQT control considering error derivative}

When the OFC is a simple first-order integrator then the cost function can only contain the tracking error and high 
order derivatives are excluded. In this variation of the LQT control the OFC is extended to include the firstorder derivative of the error function. Defining the OFC's structure as $(1+\beta s) / s$ where $\beta$ is a known positive scalar, the cost function changes to

$$
J=\int_{0}^{\infty}\left[q(e+\beta \dot{e})^{2}+v^{2}\right] d t
$$

The OFC state-space representation changes to

$$
\dot{x}_{c}=e+\beta \dot{e}=r-\boldsymbol{C} \boldsymbol{x}-\beta \boldsymbol{C}\left(\boldsymbol{A} \boldsymbol{x}+\boldsymbol{B} u+\boldsymbol{B}_{\boldsymbol{d}} d\right)
$$

And the augmented system's matrices $\boldsymbol{A}_{\boldsymbol{a}}$ and $\boldsymbol{B}_{\boldsymbol{a}}$ are transformed into

$$
\boldsymbol{A}_{a}=\left[\begin{array}{cc}
\boldsymbol{A} & 0 \\
-\boldsymbol{C}-\beta \boldsymbol{C A} & 0
\end{array}\right], \quad \boldsymbol{B}_{\boldsymbol{a}}=\left[\begin{array}{c}
\boldsymbol{B} \\
-\beta C \boldsymbol{B}
\end{array}\right]
$$

By replacing the augmented matrices and the control law defined by equation 29 in equation 25 the state-space representation is obtained.

$$
\left[\begin{array}{c}
\dot{\boldsymbol{x}} \\
\dot{x}_{c}
\end{array}\right]=\left[\begin{array}{cc}
\boldsymbol{A}-\boldsymbol{B} \boldsymbol{K}_{\boldsymbol{S}} & -\boldsymbol{B K}_{\boldsymbol{o}} \\
-\boldsymbol{C}-\beta \boldsymbol{C A}+\beta \boldsymbol{C B} \boldsymbol{K}_{\boldsymbol{s}} & \beta \boldsymbol{C B} \boldsymbol{K}_{\boldsymbol{o}}
\end{array}\right]\left[\begin{array}{l}
\boldsymbol{x} \\
x_{c}
\end{array}\right]+\left[\begin{array}{l}
0 \\
1
\end{array}\right] r+\left[\begin{array}{c}
B_{d} \\
0
\end{array}\right] d
$$

This variation of the LQT control will be named in this work as LQTB for simplicity and its solution is done as in the LQT control.

\section{Simulations}

To analyse the acceleration tracking performance of the proposed controllers, simulations were carried out using the non-linear model of the seismic shake table and using $30 \%$ of El Centro earthquake as input signal.

Fig 3 shows the acceleration tracking control of the controllers and Figure 4 shows the corresponding relative acceleration tracking error. As can be observed from both figures the tracking performance of the LQTB controller is the best among the other two controllers, presenting the smallest overall tracking error. The performance of the LQT controller is the worst, exhibiting delay error and the biggest magnitude tracking error.

In order to quantitatively compare the tracking performance of the controllers, the relative maximum error (RME), the RME index, the root mean square error (RMSE) and RMSE index are adopted for evaluation [10]. These parameters are calculated as follows

$$
\begin{gathered}
R M E=\max \left|a_{d}-a_{m}\right| \\
R M E \text { index }=\frac{\max \left|a_{d}-a_{m}\right|}{\max \left|a_{d}\right|} \cdot 100 \\
R M S E=\sqrt{\frac{1}{N} \sum_{i=1}^{N}\left(a_{d}-a_{m}\right)^{2}} \\
R M S E \text { index }=\frac{\sqrt{\frac{1}{N} \sum_{i=1}^{N}\left(a_{d}-a_{m}\right)^{2}}}{\sqrt{\frac{1}{N} \sum_{i=1}^{N}\left(a_{d}\right)^{2}}} \cdot 100
\end{gathered}
$$

where $a_{d}$ is the desired acceleration, $a_{m}$ the acceleration output of the controller and $N$ the signal length. Table 1 lists the evaluation parameters for the three controllers. These results clearly demonstrate that better acceleration tracking control can be achieved with the LQTB control than with the LQI and the conventional LQT.

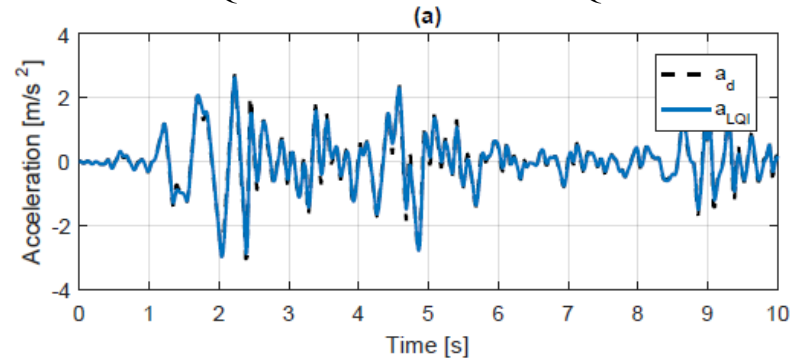

(b)

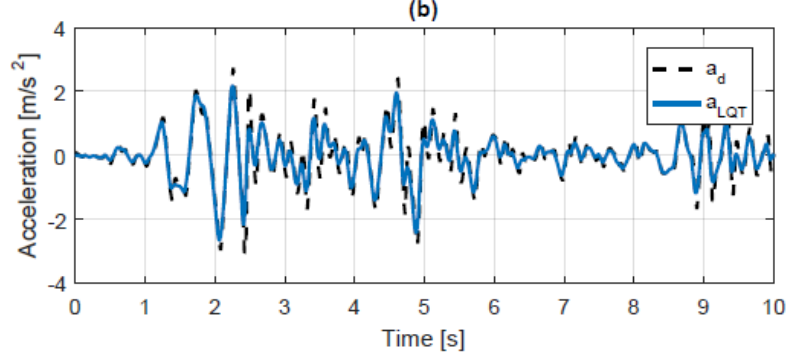

(c)

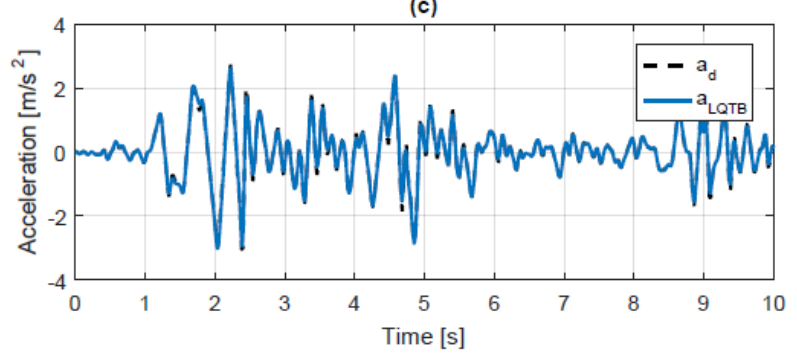

Fig 3. Acceleration tracking control with El Centro earthquake as input signal : (a) LQI control. (b) LQT control. (c) LQTB control.
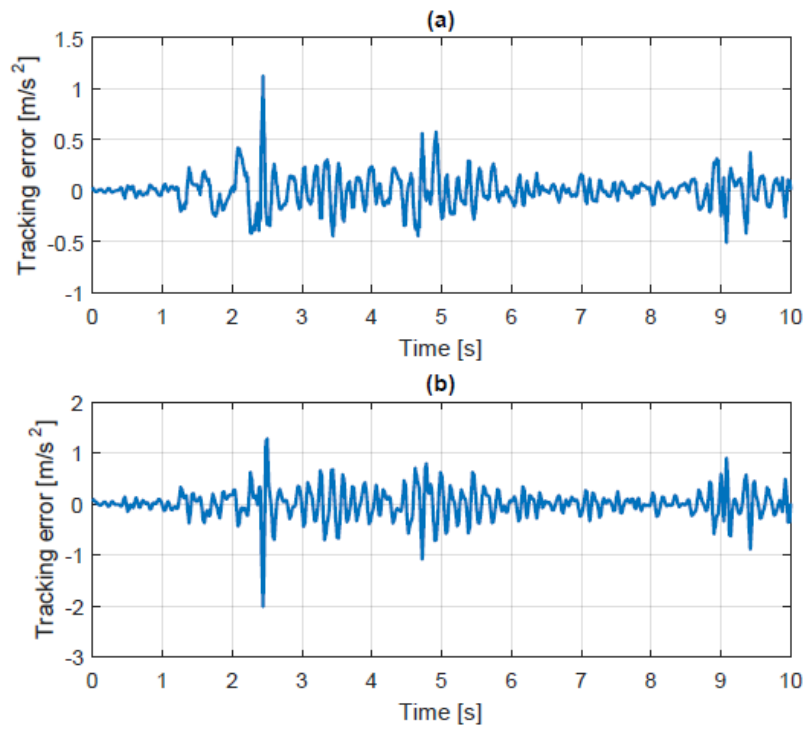

(c)

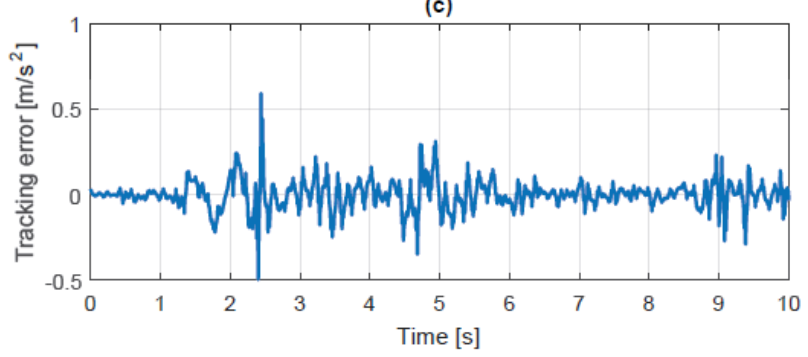

Fig 4. Tracking error with El Centro earthquake as input signal : (a) LQI control. (b) LQT control. (c) LQTB control. 
Table 1. Acceleration tracking performance of the proposed controllers for El Centro earthquake input signal.

\begin{tabular}{|c|c|c|c|c|}
\hline $\begin{array}{c}\text { Controller } \\
\text { type }\end{array}$ & $\begin{array}{c}\text { RME } \\
{\left[\boldsymbol{m} / \boldsymbol{s}^{\mathbf{2}}\right]}\end{array}$ & $\begin{array}{c}\text { RME } \\
\text { index }\end{array}$ & $\begin{array}{c}\text { RMSE } \\
{\left[\boldsymbol{m} / \boldsymbol{s}^{\mathbf{2}}\right.}\end{array}$ & $\begin{array}{c}\text { RMSE } \\
\text { index }\end{array}$ \\
\hline LQI & 1.125 & $36.93 \%$ & 0.418 & $18.52 \%$ \\
\hline LQT & 2.013 & $66.12 \%$ & 0.263 & $33.11 \%$ \\
\hline LQTB & 0.529 & $19.43 \%$ & 0.076 & $9.54 \%$ \\
\hline
\end{tabular}

Figure 5 shows the comparison of the control signal for the three controllers. As can be observed the control effort for the LQTB and LQI controllers is very similar. The control effort of the LQT is smaller due to its magnitude tracking error showed in Figu 3 (b) and 4 (b).

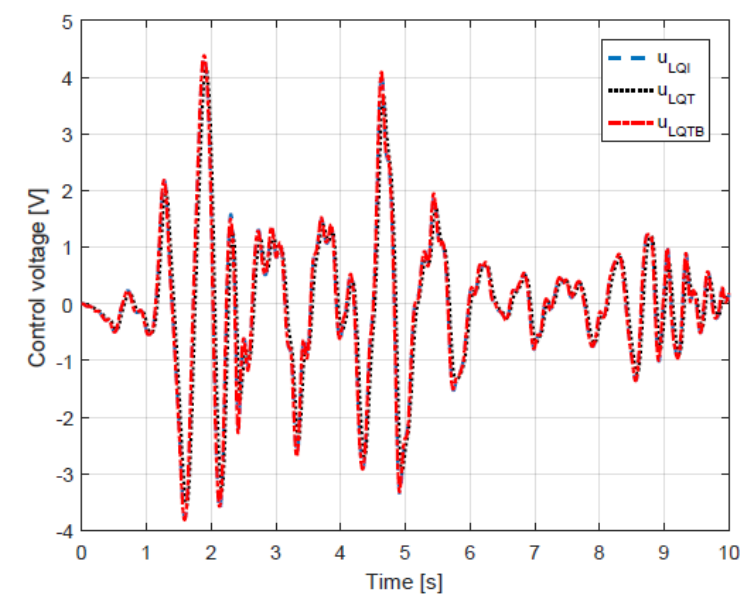

Fig 5. Control signal with El Centro earthquake input signal.

\section{Conclusions}

In this work the non-linear dynamic and mathematical model of a hydraulic uniaxial seismic shake table was developed. Three control strategies based on the LQR formulation were designed: LQI, LQT and a variation of LQT, named here LQTB. The LQI control minimizes a cost function that includes all system's states, the tracking error and the control input, while the LQT control only considers the tracking error, and the LQTB the tracking error and its derivative.

Simulations were carried out using $30 \%$ of El Centro earthquake record as the input signal. The graphic results and the evaluation parameters calculated (RME, RME index, RMSE and RMSE index) showed that the LQTB control has the best acceleration tracking performance, followed by the LQI control. The LQT controller exhibited delay error and the poorest tracking.

\section{Observations}

Future work will include experimental validation of the dynamic model and implementation of the three proposed controllers in the hydraulic uniaxial seismic shake table of the Dynamics and Structural Control Lab at Universidad Industrial de Santander (UIS). The seismic table is shown in Fig 6 and consists of a Parker hydraulic cylinder, a MOOG 76-263 servo valve and a MTS hydraulic power unit.

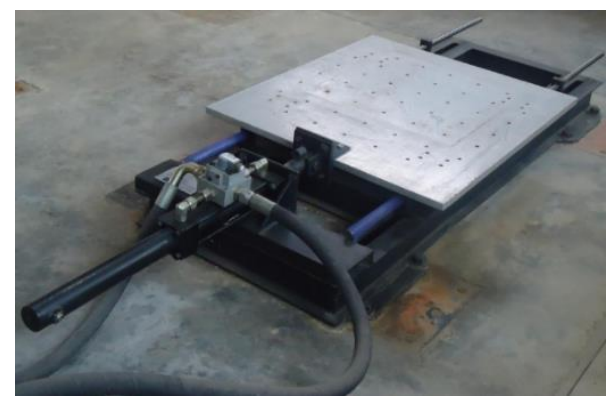

Fig 6. Unaxial seismic shake table at UIS.

\section{Acknowledgements}

This work is supported by "Vicerrectoría de Investigación (VIE)" code VIE-1366 of the Universidad Industrial de Santander, where the research project has a grant to support Master's and Bachelor's students in Mechanical Engineering, in Fluid Power research area. The funding and support of Mechanical Engineering School of Universidad Industrial de Santander is greatly appreciated.

\section{References}

1. A. R. Plummer, Control Engineering Practice, 53, 109-122 (2016)

2. R. T. Severn, D. P. Stoten, Y. Tagawa, 15th World Conference on Earthquake Engineering (2012)

3. N. Nakata, 15th World Conference on Earthquake Engineering (2012)

4. C. Borrás, J. L. Sarmiento, J. F. Ortiz, XI Congreso Colombiano de Métodos Numéricos, 6, 9 (2017)

5. J. Kuehn, D. Epp, W. N. Patten, Earthquake Engineering and Structural Dynamics, 28, 1235-1254 (1999)

6. M. Jelali, A. Kroll, Hydraulic Servo-System (Springer-Verlag, London, 2004)

7. M. Karimi-Ghartemani, S. A. Khajehoddin, P. Jain, A. Bakhshai, International Journal of Control, 84, 1442-1449 (2011)

8. U. Aimen, M. Liaquat, R. Ali, Q. Fazal, 6th International Conference on Systems and Control, 611-615 (2017)

9. Y. Tang, Z. Zhu, G. Shen, X. Li, Journal of Vibration and Control, 22, 3945-3964 (2015)

10. Y. Tang, Z. Zhu, G. Shen, W. Zhang, IEEE Access, 5, 23687-23694 (2017) 